\title{
Análise do alinhamento dos membros inferiores em crianças com excesso de peso
}

\section{Analysis of lower limb alignment in overweight children}

\author{
Alessandra Rezende Martinelli ${ }^{1}$ \\ Moisés Oliveira Purga \\ Alessandra Madia Mantovani \\ Marcela Regina Camargo \\ Alan Abrão Rosell \\ Cristina Elena Prado Teles Fregonesi ${ }^{1}$ \\ Ismael Forte Freitas Junior ${ }^{2}$
}

1 Universidade Estadual Paulista. Faculdade de Ciência e Tecnologia. Departamento de Fisioterapia. Campus de Presidente. Prudente, SP. Brasil.

2 Universidade Estadual Paulista. Departamento de Educação Física. Campus de Presidente Prudente, SP. Brasil.

Recebido em 04/12/09 Revisado em 26/07/10 Aprovado em 20/12/10
Resumo - O sobrepeso e a obesidade (SO) têm atingido proporções epidêmicas, principalmente, em idade escolar, tornando-se um problema mundial de saúde. Muitas adaptações podem ocorrer na organização postural de crianças com SO, provocando, graves consequências. A avaliação postural pode colaborar para o entendimento destas alterações. $\mathrm{O}$ objetivo do presente estudo foi verificar alterações na postura dos membros inferiores de crianças com SO, através de metodologia específica. Vinte e dois indivíduos, sendo distribuídos de acordo com o sexo: 9 do feminino e 13 do masculino, com faixa etária entre 5-9 anos, participaram do estudo. Na avaliação postural, foram utilizados a fotogrametria e o software SAPo. Foram analisados os ângulos articulares dos membros inferiores e a projeção plantar do centro de gravidade. Foi utilizada estatística não-paramétrica descritiva, além dos testes de Kruskal-Wallis e de Mann-Whitney $(\mathrm{p}<0,05)$, para comparação entre os sexos. Ambos os sexos apresentaram anteroversão e leve rotação pélvica, à direita para o feminino e à esquerda para o masculino hiperextensão e valgismo de joelhos e valgismo tornozelos. O centro de gravidade apresentou 26,95\% de anteriorização. Conclui-se que a avaliação postural nessa população foi congruente aos achados da literatura e a metodologia utilizada possibilitou quantificar os dados para futuras comparações.

Palavras-chave: Obesidade; Postura; Desenvolvimento infantil.

Abstract - Overweight and obesity have reached epidemic proportions, especially among schoolchildren, and have become a global health problem. Many adaptations in postural organization are observed in overweight and obese children, which have serious consequences. Posture assessment contributes to the understanding of these changes. The aim of this study was to assess changes in lower limb posture of overweight and obese children using a specific method. Twenty-two subjects, 9 girls and 13 boys, aged 5-9 years participated in the study. Posture was assessed by photogrammetry using the SAPo software. The lower limb joint angles and plantar projection of the center of gravity were analyzed. Nonparametric and descriptive statistics were used, in addition to the Kruskal-Wallis and Mann-Whitney tests $(p<0.05)$ for comparison between genders. Both groups showed anterior pelvic tilt and slight rotation (to the right in girls and to the left in boys), hyperextension, and valgus knees and ankles. The center of gravity presented $26.95 \%$ anteriorization. In conclusion, posture assessment in this population was consistent with literature findings and the method used permitted the quantification of data for future comparisons. Key words: Obesity; Posture; Child development. 


\section{INTRODUÇÃO}

O sobrepeso e a obesidade ( $\mathrm{SO}$ ) têm atingido proporções epidêmicas, principalmente em idade escolar $^{1,2}$, e já assumem frequência de maior magnitude que a desnutrição ${ }^{3}$. Em algumas regiões brasileiras, a prevalência de $\mathrm{SO}$ em crianças com idade inferior a 10 anos pode ultrapassar os $40 \% 4,5$, tornando o fato um problema de saúde pública ${ }^{6}$. SO são transtornos multifatoriais e estão associados ao nível de atividade física? ${ }^{7}$ A relação causa e consequência de tais transtornos ainda não estão bem elucidadas ${ }^{8}$, no entanto, sabe-se que fatores como uma alta ocorrência de fraturas e desconforto musculoesquelético, pouca mobilidade geral e o mau alinhamento do membro inferior são mais frequentes em crianças com $\mathrm{SO}$ e colaboram para a perpetuação do acúmulo de gordura9.

Embora sejam poucos os estudos específicos sobre o impacto da obesidade no sistema musculoesquelético infantil, Wearing et. al ${ }^{10}$ descrevem que muitas adaptações podem ocorrer na organização postural dessas crianças, como alterações no alinhamento e na estrutura dos quadris, joelhos, tornozelos e pés, provocando dor, desconforto, ineficiência da biomecânica corporal e consequente redução da mobilidade. Ademais, crianças com SO apresentam maior dificuldade de controle postural, possivelmente, influenciada pela deficitária propriocepção nos joelhos, comumente observada ${ }^{11,12}$.

Grande parte dos problemas posturais desenvolvidos durante a infância permanece na vida adulta. Portanto, a crescente incidência de obesidade infantil, que aumenta a predisposição aos desvios posturais, pode resultar em uma população adulta com membros inferiores significativamente desalinhados ${ }^{13}$. Para que isso não ocorra, é necessário que se entenda um pouco melhor como o SO desfavorecem o desenvolvimento adequado dos membros inferiores. A avaliação postural pode ser a chave para esse entendimento.

Ainda há muita discussão acerca da utilização desse recurso, devido à diversidade de técnicas disponíveis e à discordância entre os padrões de normalidade encontrados, contudo, é consenso que uma postura equilibrada é importante para um bom funcionamento das estruturas musculoesqueléticas ${ }^{14}$. Estudos atuais que utilizam a avaliação da postura através da análise de fotografias têm apresentado boa confiabilidade dos dados e reprodutibilidade da técnica ${ }^{14,15}$. Dessa maneira, parece que a fotogrametria surgiu como um alento para as investigações posturais e vem se consolidando e se tornando consenso entre os pesquisadores.
De uma maneira geral, é encontrada na literatura certa quantidade de estudos que pesquisam o posicionamento dos membros inferiores de crianças obesas, contudo, sem sua devida quantificação $0^{11-13,15}$. Ademais, grande parte deles utiliza métodos cujos resultados não são padronizados e variam de acordo com o instrumento utilizado ${ }^{10}$. O fato de a fotogrametria vir sendo amplamente aceita para avaliação da postura aliada ao pressuposto de que os aspectos do SO possam influenciar na biomecânica postural, o presente estudo buscou avaliar, descrever e comparar os sexos e hemicorpos, com a utilização da fotogrametria e de um software específico, o padrão postural dos membros inferiores de crianças com excesso de gordura corporal.

\section{PROCEDIMENTOS METODOLÓGICOS}

\section{Sujeitos da Pesquisa}

Foram avaliadas 22 crianças com SO e idade entre cinco e nove anos, de ambos os sexos, provenientes do Projeto de Extensão Universitária "Programa Super Ação", da Faculdade de Ciências e Tecnologia da Universidade Estadual Paulista (FCT/ UNESP). O estado nutricional foi classificado pelo índice de massa corporal (IMC), seguindo valores de referência para sexo e idade, publicados por Cole et. $\mathrm{al}^{16}$. Os critérios de inclusão neste estudo foram o enquadramento do participante na categoria sobrepeso ou obesidade, a ausência de enfermidades de ordem neurológica, psíquica ou outros problemas médicos graves que pudessem alterar a postura.

Este estudo foi elaborado de acordo com os Critérios da Ética em Pesquisa com Seres Humanos (Resolução 196/96 do Conselho Nacional de Saúde) e aprovado pelo Comitê de Ética em Pesquisa da FCT/UNESP (205/2007).

\section{Procedimentos}

A coleta dos dados foi realizada no Laboratório de Estudos Clínicos em Fisioterapia (LECFisio) da FCT/UNESP, em diversos horários do dia, de acordo com a disponibilidade dos participantes. A temperatura ambiente foi mantida em $21^{\circ} \mathrm{C}$ e as crianças foram orientadas a não praticar nenhum tipo de atividade desgastante anteriormente à coleta. Num primeiro momento, foram registradas a idade, massa e estatura dos indivíduos para cálculo do IMC.

Em seguida, foi iniciada a avaliação da postura por meio de fotogrametria com utilização do Software de Avaliação Postural (SAPo) ${ }^{17}$, ferramenta validada, confiável e reprodutível (coeficiente de correlação intra-classe de $0,99^{15}$ ). Para isso, o ambiente e o par- 
ticipante foram preparados. Uma câmera digital foi posicionada sobre um tripé, a uma altura correspondente a 50\% da estatura do indivíduo e a uma distância de 3,00m do mesmo. No participante, orientado a trajar roupas de banho, foram fixados com fita adesiva dupla-face, marcadores de isopor, sobre alguns dos pontos anatômicos pré-determinados pelo protocolo SAPo (Figura 1). Em seguida, foi solicitado ao indivíduo que permanecesse em posição ortostática, paralelamente a um fio de prumo fixado ao teto, de tal modo que este fio e o avaliado ficassem em um mesmo plano, perpendicular ao eixo da câmera. Para permitir uma posterior calibração no software, foram colocadas esferas de isopor no fio de prumo, a uma distância conhecida de $0,40 \mathrm{~m}$ entre elas.

Após o posicionamento do indivíduo, foram feitas 12 imagens: três em vista anterior, três em posterior, três laterais esquerdas e três direitas. Esse procedimento garantiu que fossem realizadas médias entre as imagens em cada vista, evitando-se possíveis erros casuais. Para manter a mesma base de sustentação, foi utilizado um tapete preto de borracha, no qual o indivíduo se posicionou livremente em vista anterior, para a primeira fotografia. Neste momento, os pés foram contornados com giz, visando direcionar a posição dos mesmos nas imagens subsequentes. Assim, o tapete foi rodado $90^{\circ}$ da posição inicial para cada nova foto. Ao sujeito foi solicitado sempre posicionar seus pés sobre o desenho, inicialmente, feito no tapete. $O$ posicionamento do tapete foi padronizado dentro de uma moldura de 0,50 ×0,50m, marcada no solo com fita adesiva.

Posteriormente, foi realizada a calibração das imagens, com a qual foi ajustada a direção vertical da foto e as distâncias na imagem foram transformadas de pixels para distâncias reais do objeto (medidas em metro). Após a calibração, os marcadores foram identificados e delimitados na fotografia.
Assim, as imagens digitalizadas foram analisadas pelo software que determinou os ângulos articulares e a projeção plantar do centro de gravidade (CG).

\section{Descrição das variáveis}

Nas análises das imagens digitalizadas pelo software SAPo, angulações com valores negativos (-) indicam desvios à esquerda $(\mathrm{E})$ no plano frontal (vista anterior e posterior) e desvio posterior no plano sagital (vista lateral esquerda e direita); já angulações com valores positivos (+) indicam desvios à direita (D) no plano frontal e desvio anterior no plano sagital. As medidas analisadas e suas respectivas vistas; siglas e descrições podem ser observadas no quadro 1.

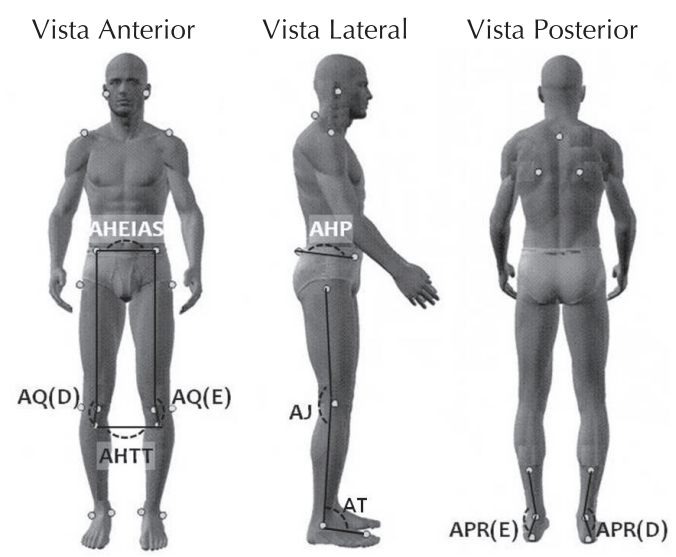

Figura 1. Pontos pré-determinados pelo protocolo SAPo e descrição dos ângulos analisados pelo software e utilizados no estudo.

Notas - AHEIAS: Alinhamento horizontal das espinhas ilíacas ântero-superiores; AHTT: Alinhamento horizontal das tuberosidades tibiais; AQ: Ângulo do quadril; AHP: Alinhamento horizontal da pelve; AJ: Alinhamento do joelho; AT:Alinhamento do tornozelo; APR: Alinhamento entre a perna e o retropé. D: Direita; E: Esquerda.

Em relação à projeção plantar do $\mathrm{CG}$, sua estimativa é baseada em cálculos específicos já

Quadro 1. Vista, sigla e descrição dos ângulos avaliados pelo protocolo do SAPo.

\begin{tabular}{|c|c|c|c|}
\hline Vista & Medida & Sigla & Descrição \\
\hline \multirow{3}{*}{ Anterior } & $\begin{array}{l}\text { Alinhamento horizontal entre } \\
\text { as espinhas ilíacas ântero-superiores. }\end{array}$ & AHEIAS & $\begin{array}{c}\text { Relação entre as espinhas ilíacas ântero-superiores direita } \\
\text { e esquerda e a horizontal. }\end{array}$ \\
\hline & $\begin{array}{l}\text { Alinhamento horizontal } \\
\text { das tuberosidades tibiais. }\end{array}$ & AHTT & Relação entre as tuberosidades tibiais e a horizontal. \\
\hline & Ângulo do quadril direito / esquerdo. & $\begin{array}{l}A Q(D) e \\
A Q(E)\end{array}$ & $\begin{array}{c}\text { Ângulo entre a espinha ilíaca ântero-superior, o centro da } \\
\text { patela e a tuberosidade tibial. }\end{array}$ \\
\hline Posterior & $\begin{array}{l}\text { Alinhamento entre a perna e o retropé } \\
\text { direito / esquerdo. }\end{array}$ & $\begin{array}{l}\operatorname{APR}(D) \text { e } \\
\operatorname{APR}(E)\end{array}$ & $\begin{array}{c}\text { Ângulo entre a linha média da perna, o ponto médio } \\
\text { entre os maléolos e o calcâneo. }\end{array}$ \\
\hline \multirow{3}{*}{ Lateral } & $\begin{array}{l}\text { Alinhamento horizontal da pelve } \\
\text { direito / esquerdo. }\end{array}$ & $\mathrm{AHP}(\mathrm{D})$ e $(\mathrm{E})$ & $\begin{array}{l}\text { Relação entre a espinha ilíaca ântero e póstero-superior } \\
\text { e a horizontal. }\end{array}$ \\
\hline & $\begin{array}{l}\text { Alinhamento do joelho direito / } \\
\text { esquerdo. }\end{array}$ & $A J(D)$ e $(E)$ & $\begin{array}{c}\text { Ângulo entre o trocânter maior do fêmur, linha articular } \\
\text { do joelho e maléolo lateral. }\end{array}$ \\
\hline & $\begin{array}{l}\text { Alinhamento do tornozelo direito / } \\
\text { esquerdo. }\end{array}$ & $\mathrm{AT}(\mathrm{D})$ e $(\mathrm{E})$ & $\begin{array}{c}\text { Ângulo entre a linha articular do joelho, maléolo lateral e } \\
\text { a horizontal. }\end{array}$ \\
\hline
\end{tabular}


embutidos no SAPo e as medidas são realizadas através da análise da distância entre a projeção do CG e a base de suporte no plano sagital, com origem na posição média entre os dois maléolos, descritos em porcentagem (\%).

\section{Análise dos dados}

Para análise dos resultados, inicialmente, foi aplicado o teste Kolmogorov-Smirnov-Z, e verificou-se uma distribuição não-normal dos dados. Sendo assim, optou-se pela utilização da estatística não-paramétrica com as medidas descritivas: divisão em quartis e amplitudes, além da distribuição de frequências. Foram aplicados, também, os testes $U$ de Mann-Whitney e H de Kruskal-Wallis, para análise das variáveis entre os sexos e hemicorpos, respectivamente, com $\mathrm{p}<0,05$. Para realização do cálculo estatístico, foi utilizado o software SPSS versão 13.0.

\section{RESULTADOS}

As características referentes à idade, estatura, massa e ao IMC dos participantes estão expressos na Tabela 1. De acordo com Cole et. $a^{16}$, todos os participantes foram considerados obesos.

As medianas e amplitudes referentes à avaliação das amplitudes e dos alinhamentos estudados estão na Figura 2.

Em vista anterior, os valores do AHEIAS e do AHTT apresentaram-se opostos entre os sexos feminino e masculino. O sexo feminino apresentou ligeira rotação pélvica e aumento do AHTT à D, enquanto o masculino apresentou ligeira rotação pélvica e um aumento do AHTT à E. Em vista lateral, os valores de AHP indicaram que ambos os sexos apresentaram algum grau de anteroversão pélvica e, em relação ao $\mathrm{AJ}$ e ao AT, houve, em geral, algum grau de hiperextensão de joelhos e pouca dorsiflexão de tornozelos em ambos os sexos. Em vista posterior, os valores do APR indicam que meninos e meninas apresentaram valores próximos e similares, demonstrando valgismo de tornozelo, em ambos hemicorpos.
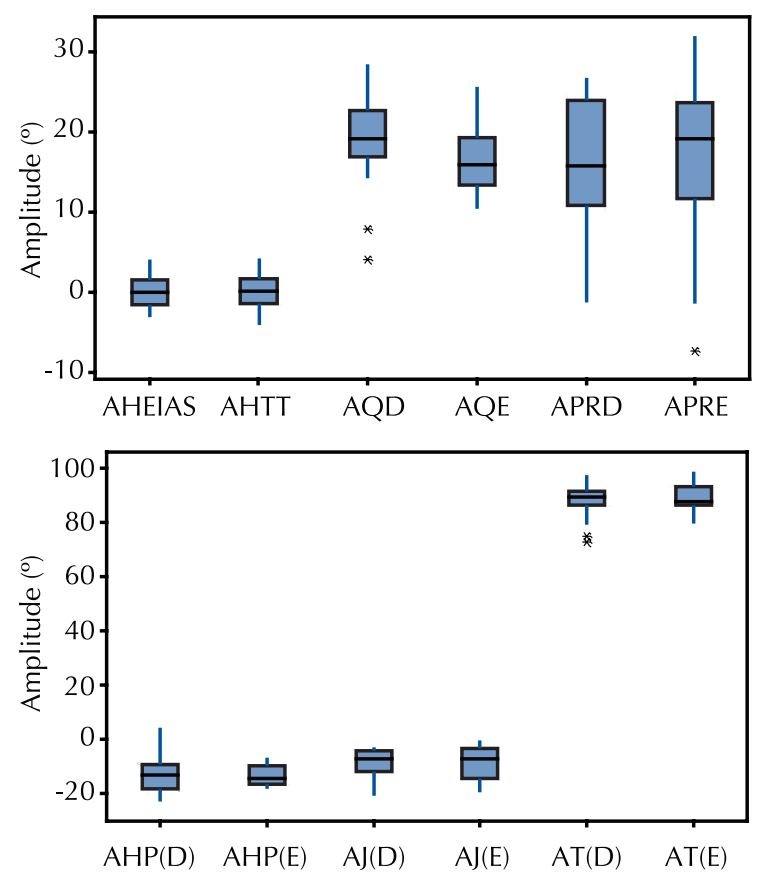

Figura 2. Medianas e amplitudes referentes à avaliação da postura dos membros inferiores. (2A - Variáveis do plano anterior e posterior e 2B - Variáveis do plano lateral), para a amostra total do estudo. $(n=22)$.

Notas: AHEIAS: Alinhamento horizontal entre as espinhas ilíacas ântero-superiores; AHTT: Alinhamento entre as tuberosidades tibiais; AQ: Ângulo do quadril; APR: Alinhamento entre perna e o retropé; AHP: Alinhamento horizontal da pelve; AJ: Alinhamento do joelho; AT: Alinhamento do tornozelo. D: Direita; E: Esquerda. *Outliers. $\mathrm{n}=22$

Tabela 1. Valores das medianas e amplitudes referentes à idade, estatura e ao IMC da amostra total e separada por sexo. ( $\mathrm{n}=22)$

\begin{tabular}{lcccc}
\hline & Idade $($ anos $)$ & Estatura $(\mathrm{m})$ & Massa $(\mathrm{kg})$ & $\mathrm{IMC}\left(\mathrm{kg} / \mathrm{m}^{2}\right)$ \\
\hline Feminino $(\mathrm{n}=9)$ & $8(5-9)$ & $1,37(1,09-1,56)$ & $44,05(26,30-69,00)$ & $26,6(21,80-29,60)$ \\
Masculino $(\mathrm{n}=13)$ & $8(5-9)$ & $1,40(1,18-1,55)$ & $52,80(31,80-73,40)$ & $25,60(22,80-30,70)$ \\
Total & $8(5-9)$ & $1,37(1,09-1,56)$ & $50,70(26,30-73,40)$ & $25,75(21,80-30,70)$ \\
\hline
\end{tabular}

Tabela 2. Estatística e p-valor para comparação entre os sexos ( $M x \mathrm{~F}$ - Teste Mann-Whitney) e para comparação entre os hemicorpos (D $x \mathrm{E}$ - Teste Kruskal-Wallis).

\begin{tabular}{|c|c|c|c|c|c|c|c|c|c|c|c|c|}
\hline \multirow{2}{*}{ Teste Estatístico } & \multirow{2}{*}{ AHEIAS } & \multirow{2}{*}{ AHTT } & \multicolumn{2}{|c|}{$\mathrm{AQ}$} & \multicolumn{2}{|c|}{ APR } & \multicolumn{2}{|c|}{$\mathrm{AHP}$} & \multicolumn{2}{|c|}{ AJ } & \multicolumn{2}{|c|}{ AT } \\
\hline & & & $\mathrm{D}$ & $E$ & $\mathrm{D}$ & $\mathrm{E}$ & $\mathrm{D}$ & $\mathrm{E}$ & D & $\mathrm{E}$ & D & $\mathrm{E}$ \\
\hline $\begin{array}{l}\text { Mann-Whitney } \\
\text { (Grupos) }\end{array}$ & 40,50 & 48,00 & 31,50 & 42,50 & 52,00 & 57,00 & 40,50 & 53,00 & 38,50 & 51,00 & 43,50 & 52,00 \\
\hline $\mathrm{p}$-valor & 0,22 & 0,48 & 0,07 & 0,28 & 0,66 & 0,92 & 0,22 & 0,71 & 0,18 & 0,61 & 0,31 & 0,66 \\
\hline $\begin{array}{l}\text { Kruskal-Wallis }\left(\chi^{2}\right) \\
\text { (Hemicorpos) }\end{array}$ & - & - & \multicolumn{2}{|c|}{4,72} & \multicolumn{2}{|c|}{0,37} & \multicolumn{2}{|c|}{0,03} & \multicolumn{2}{|c|}{0,33} & \multicolumn{2}{|c|}{0,12} \\
\hline $\mathrm{p}$-valor & - & - & \multicolumn{2}{|c|}{$0,03^{*}$} & \multicolumn{2}{|c|}{0,54} & \multicolumn{2}{|c|}{0,85} & \multicolumn{2}{|c|}{0,56} & \multicolumn{2}{|c|}{0,73} \\
\hline
\end{tabular}

M: masculino; F: feminino; D: Direita; E: Esquerda; AHEIAS: Alinhamento horizontal entre as espinhas ilíacas ântero-superiores; AHTT: Alinhamento entre as tuberosidades tibiais; AQ: Ângulo do quadril; AHP: Ângulo horizontal da pelve; APR: Alinhamento entre perna e o retropé; AJ: Alinhamento do joelho; AT: Alinhamento do tornozelo. ${ }^{*} p<0,05$. 
Embora grande parte das variáveis coletadas não tenha sido numericamente semelhante entre os grupos e entre os hemicorpos, após a aplicação do Teste Mann-Whitney e Kruskal-Wallis (Tabela 2), respectivamente, não houve diferença significante entre eles (exceção para $\mathrm{AQ}$, quando comparados os hemicorpos).

No total da amostra estudada, o CG demonstrou-se em valores medianos (amplitude) 26,95\% (7,60-64,40\%) anteriorizado. Para os grupos, os valores foram 22,20\% (7,60-60,80\%) no GM e $32,70 \%(15,50-64,40 \%)$ no GF.

Com o objetivo de sintetizar os resultados, foi realizada uma distribuição de frequências dos principais desvios posturais decorrentes das alterações angulares em todos os planos, dentro de cada grupo e na amostra total, que está exposta na Figura 3. Os valores da pelve foram padronizados de acordo com Mac-Thiong et al. ${ }^{18}$; dos joelhos, em vista sagital, foram padronizados de acordo com Bird ${ }^{19}$ e, em vista frontal, de acordo com Neves e Campagnolo ${ }^{20}$ e os de tornozelos foram padronizados de acordo com Malhotra ${ }^{21}$ e Hennessy ${ }^{22}$.

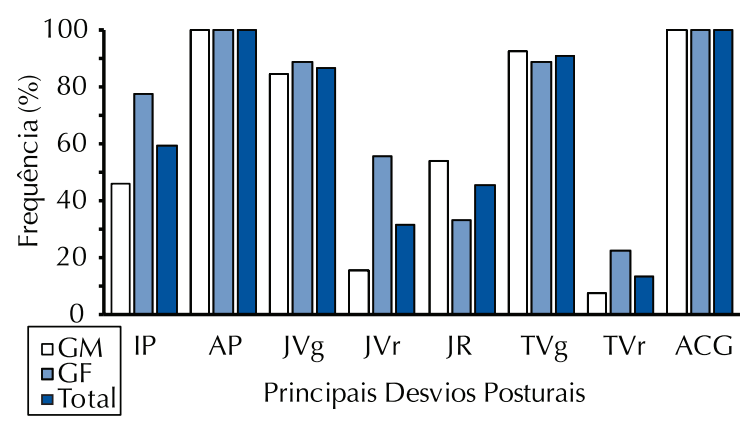

Figura 3. Proporção de indivíduos com desvios posturais. IP: Inclinação pélvica; AP: Anteroversão pélvica; JVg: Genu Valgum; JVr: Genu Varum; JR: Genu Recurvatum; TVg: Tornozelo valgum; TVr: Tornozelo varum; ACG: Anteriorização do centro de gravidade; GM: Grupo masculino $(n=13)$; GF: Grupo feminino $(n=9)$; Total: $n=22$.

\section{DISCUSSÃO}

alinhamento postural no plano sagital muda consideravelmente entre as idades de 4-12 anos, o que engloba a faixa etária do presente estudo (5-9 anos). Essas mudanças, decorrentes da maturação fisiológica do sistema musculoesquelético, possivelmente ocorrem para evitar um inadequado deslocamento anterior do $\mathrm{CG}^{23}$. Quando existe excesso de peso, os desvios posturais tentam compensar as alterações na pelve também ocasionadas pela anteriorização do $\mathrm{CG}^{24}$. O presente estudo mostrou resultados que corroboram essa proposição, uma vez que a projeção do CG encontrou-se anteriorizada, possível causa das alterações posturais encontradas.
Segundo Kendall et al. ${ }^{25}$, o alinhamento pélvico é a chave para a qualidade do alinhamento postural global. Na ocorrência de desequilíbrios dessa estrutura, a postura, como um todo será afetada. A posição fisiológica da pelve conduz a um bom posicionamento do abdome, tronco e membros inferiores. Os resultados deste estudo demonstraram que, em relação ao AHP(D) e AHP(E), 100\% da amostra apresentou algum grau de báscula anterior da pelve. Possivelmente, em consequência de tal fato, na análise sagital dos joelhos foi observado que 45,45\% da amostra apresentaram genu recurvatum, e sua análise frontal mostrou valores iguais a $19,15^{\circ}$ $(\mathrm{AQ}(\mathrm{D}))$ e $15,90^{\circ}(\mathrm{AQ}(\mathrm{E}))$, resultando em valgismo em ambos joelhos. Em relação aos tornozelos, os valores de $\operatorname{APR}(\mathrm{D})\left(15,85^{\circ}\right)$ e $\operatorname{APR}(\mathrm{E})\left(19,20^{\circ}\right)$, indicariam também, a presença de valgismo. A obesidade pode levar a desequilíbrios nas articulações dos pés e pequenas alterações em sua estrutura ou em sua disposição em relação à articulação do tornozelo, o que pode causar influências nas estratégias de controle postural, bem como no próprio alinhamento das demais articulações dos membros inferiores e tronco. No plano frontal, o valgismo de tornozelos tem sido relacionado ao dos joelhos e ambos à obesidade e ao desabamento do arco longitudinal plantar ${ }^{13}$.

O estudo de Campos et al. ${ }^{26}$, com adolescentes obesos e avaliação por simetrógrafo apóia grande parte dos presentes resultados, uma vez que 92\% das meninas e 69\% dos meninos apresentaram anteroversão pélvica; 49\% do total apresentaram hiperextensão de joelhos; $50 \%$ e 13\% das meninas e $38 \%$ e $23 \%$ dos meninos apresentaram genu valgum e genu varum, respectivamente. Resultados concordantes também foram encontrados no estudo de Pinto et. all ${ }^{27}$, com pré-adolescentes obesos e avaliação clínica, no qual, foi observado genu recurvatum em 24,40\% e genu valgum em 55,10\% da amostra. $\mathrm{O}$ estudo de Cicca et al. ${ }^{13}$, que utilizou fotogrametria para avaliar crianças obesas, corrobora, parcialmente, os presentes achados, uma vez que foram observados genu valgum em 52\% da amostra, todavia, em relação ao APR, apesar dos ângulos de crianças obesas $\left(7,97 \pm 3,99^{\circ}\right)$ e eutróficas $\left(6,06 \pm 5,91^{\circ}\right)$ terem sido diferentes, o valgismo não foi evidente.

As diferentes proporções entre os desvios de cada articulação, quando se compara a presente amostra com outros estudos, talvez possa ser justificada pela diferente média de idade, IMC e também por diferentes critérios de referências adotados por cada autor. Mesmo assim, parece evidente que o 
excesso de gordura corporal pode acentuar a anteroversão da pelve como descrito na literatura ${ }^{10,28}$; as adaptações do joelho tendem à presença de valgismo de joelhos nessa população $0^{10,13,24,28}$, embora o varismo também seja encontrado; o desabamento do arco plantar contíguo ao valgismo do tornozelo, também parece ser um achado comum.

Ainda que não tenham sido encontradas diferenças entre grupos e hemicorpos (com uma exceção), pouco foi encontrado em relação a esses parâmetros, pois grande parte das pesquisas atribui a eles papel de covariáveis, não os comparando. A diferença encontrada entre o $\mathrm{AQ}(\mathrm{D})$ e o $\mathrm{AQ}(\mathrm{E})$, não foi observada no estudo de Cicca et al..$^{13} \mathrm{e}$ talvez seja derivada de possíveis escolioses, deformidade comum em crianças nessa faixa etária ${ }^{29}$. O desarranjo da estrutura axial pode ter levado a assimetrias das articulações apendiculares, desencadeando tal diferença. Desta maneira, esse achado provavelmente não esteja relacionado à obesidade.

De uma maneira geral, os indivíduos aqui avaliados apresentaram, então, valgismo dos tornozelos, hiperextensão dos joelhos, rotação medial de quadris e anteroversão pélvica, o que pode ter provocado o deslocamento anterior do CG. As demais variáveis coletadas (AHEIAS, AHTT e AT) mostraram alterações que possivelmente se relacionem aos distúrbios já discutidos, ou possam ser consideradas alterações de menor importância e mais relativas à variedade individual ou à própria fase de crescimento ${ }^{30}$.

Embora os resultados encontrados possuam valor respaldado cientificamente ${ }^{13-15,17,29}$, é necessário levar em consideração algumas limitações, como a falta de um grupo eutrófico, o que não tornou possível a realização de comparações sobre as incidências dos desvios posturais, prejudicando um estudo mais aprofundado sobre a condição.

\section{CONCLUSÃO}

Com a avaliação, pôde-se observar um padrão postural semelhante ao descrito na literatura no qual são incluídos valgismo dos tornozelos e joelhos, além de sua hiperextensão, rotação medial de quadris e anteroversão pélvica. Não foi observada diferença desse padrão entre os sexos e nem entre os hemicorpos, quando comparados estatisticamente. Foi possível quantificar e assinalar os principais desvios e desalinhamentos articulares através de uma ferramenta específica para tal finalidade, confiável e com reprodutibilidade aceitável. Futuros estudos, com a mesma metodologia e inserção de um grupo controle, devem ser incentivados no sentido de se obter parâmetros padronizados de comparação.

\section{REFERÊNCIAS BIBLIOGRÁFICAS}

1. McDonald CM, Baylin A, Arsenault JE, Mora-Plazas $\mathrm{M}$, Villamor E. Overweight is more prevalent than stunting and is associated with socioeconomic status, maternal obesity, and a snacking dietary pattern in school children from Bogota, Colombia. J Nutr 2009;139(2):370-6.

2. Bergmann GG, Bergmann MLA, Pinheiro ES, Moreira RB, Marques AC, Garlipp DC et al. Índice de massa corporal: tendência secular em crianças e adolescentes brasileiros. Rev Bras Cineantropom Desempenho Hum 2009;11(3):280-5.

3. Fernandes RA, Kawaguti SS, Agostini L, Oliveira AR, Ronque RV, Freitas Júnior IF. Prevalência de sobrepeso e obesidade em alunos de escolas privadas do município de Presidente Prudente-SP. Rev Bras Cineantropom e Desempenho Hum 2007;9(1):21-7.

4. Costa RF, Cintra IP, Fisberg M. Prevalência de sobrepeso e obesidade em escolares da cidade de Santos-SP. Arq Bras Endocrinol Metab 2006;50(1):60-7.

5. Brasil LMP, Fisberg M, Maranhão HS. Excesso de peso em uma região do nordeste brasileiro: contraste entre as redes de ensino pública e privada. Rev Bras Saúde Matern Infant 2007;7(4):405-12.

6. Bozza R, Neto AS, Ulbrich AZ, Vasconcelos IQPG, Mascarenhas LPG, Brito LMS et al. Circunferência da cintura, índice de massa corporal e fatores de risco cardiovascular na adolescência. Rev Bras Cineantropom Desempenho Hum 2009;11(3):286-91.

7. Januário RSB, Nascimento MA, Barazetti LK, Reichert FF, Mantoan JPB, Oliveira AR. Índice de massa corporal e dobras cutâneas como indicadores de obesidade em escolares de 8 a 10 anos. Rev Bras Cineantropom Desempenho Hum 2008;10(3):266-70.

8. Matsudo VKR, Matsudo SMM. Atividade física no tratamento da obesidade. Einstein 2006;4(supl.1):S29-S43.

9. Taylor ED, Theim KR, Mirch MC, Ghorbani S, Tanofsky-Kraff M, Adler-Wailes DC et al. Orthopedic complications of overweight in children and adolescents. Pediatrics 2006;117(6):2167-74.

10. Wearing SC, Hennig EM, Byrne NM, Steele Jr, Hills AP. The impact of childhood obesity on musculoskeletal form. Obes Rev 2006;7(2):209-18.

11. D'Hondt E, Deforche B, Bourdeaudhuij I, Lenoir, M. Childhood obesity affects fine motor skill performance under different postural constraints. Neurosci Lett 2008;440(1):72-5.

12. Wang L, Li JX, Xu DQ, Hong YL. Proprioception of ankle and knee joints in obese boys and nonobese boys. Med Sci Monit 2008;149(3):129-35.

13. Cicca LO, João SMA, Sacco ICN. Caracterização postural dos membros inferiores de crianças obesas de 7-10 anos. Fisioter Pesqui 2007;14(2):40-6. 
14. Sacco ICN, Aliberti S, Queiroz BWC, Pripas D, Kieling I, Kimura AA et al. Confiabilidade da fotogrametria em relação à goniometria para avaliação postural de membros inferiores. Rev Bras Fisioter 2007;11(5):411-7.

15. Braz RG, Goes FPC, Carvalho GA. Confiabilidade e validade de medidas angulares por meio do software para avaliação postural. Fisioter Mov 2008;21(3):117-26.

16. Cole TJ, Bellizzi MC, Flegal KM, Dietz WH. Establishing a standard definition for child overweight and obesity worldwide: international survey. BMJ 2000;320:1-6.

17. Duarte M, Maldonado EP, Freitas AZ, Ferreira EA, Prado J, Pasqual AP, et al. Software para Avaliação Postural. São Paulo: Universidade de São Paulo, 2006. Avaliable from: <http://sapo.incubadora.fapesp.br> [2010 sep 14].

18. Mac-Thiong JM, Berthonnaud E, Dimar JR, Betz RR, Labelle HMD. Sagittal alignment of the spine and pelvis during growth. Spine 2004(29):15:1642-7.

19. Bird HA. Joint hypermobility. Reports from interest groups of annual general meeting of the british society for rheumatology. Br J Rheumatol. 1992;31(3):205-8.

20. Neves CM, Campagnolo JL. Desvios axiais dos membros inferiores. Rev Port Clin Geral 2009:25(4):464-70.

21. Malhotra D, Puri R, Owen R. Valgus deformity of the ankle in children with spina bifida aperta. J Bone Joint Surg Br 1984;66(3):381-5.

22. Hennessy MS, Molloy AP, Wood EV. Management of the varus arthritic ankle. Foot Ankle Clin 2008;13(3):417-42.

23. Lafond D, Descarreaux M, Normand MC, Harrison DE. Postural development in school children: a cross-sectional study. Chiropr Osteopat 2007; 15(1) Avaliable from: $<$ http://www.chiroandosteo.com/content/15/1/1> [2010 sep 14].
24. Bruschini S, Nery CAS. Aspectos ortopédicos da obesidade na infância e adolescência. In: Fisberg M. Obesidade na infância e adolescência. São Paulo: Fundação Editorial BYK;1995,p.105-125.

25. Kendall PF, Mccreay EK, Provance PG. Músculos provas e funções. 4a ed. São Paulo: Manole;1995.

26. Campos FL, Silva AS, Fisberg M. Descrição fisioterapêutica das alterações posturais de adolescentes obesos. Braz Pedriatr News [periódico on-line] 2002. <http:// www.portalsaudebrasil.com/artigospsb/obes167.pdf> [2010 sep 14]

27. Pinto ALS, Holanda PMB, Radu AS, Villares SMF, Lima FR. Musculoskeletal findings in obese children. J Paediatr Child Health 2006;42(6):341-4.

28. Reilly JJ, Methven E, Mcdowell ZC, Hacking B, Alexander D, Stewart $\mathrm{L}$ et al. Health consequences of obesity. Arch Dis Child 2003;88(9):748-52.

29. Kussuki MOM, João SMA, Cunha ACP. Caracterização postural da coluna de crianças obesas de 7 a 10 anos. Fisioter Mov 2007;20(1):77-84.

30. Krul M, van der Wouden JC, Schellevis FG, van Suijlekom-Smit LW, Koes BW. Musculoskeletal problems in obese children. Ann Fam Med 2009;7(4):352-6.

\section{Endereço para correspondência}

Alessandra Rezende Martinelli.

Rua: José Soares Filho, 460. Parque Francal. CEP: 14403-128, Franca, São Paulo, Brasil. E-mail: lerezendemartinelli@yahoo.com.br 